\title{
Distribución de los activos monetarios del sector privado en El Salvador
}

Carlos J. Glower

A la memoria del maestro Jorge Sol Casiellanos, creador de instituciones, diseminador de ciencia.

\section{Introducción}

Para propósitos didácticos usualmente se senalan tres elementos básicos que contribuyen a delerminar la oferta monetaria, en una economia moderna. En primer lugar, se debe mencionar el papel que juega la autoridad monelaria al ejercer conitrol direclo sobre la base monetaria.' Un segundo elemenlo lo representa el sector bancario, que trata de minimizar la diterencial entre las reservas obligalorias depositadas en el banco central y los fondos ociosos. Por su parte, el tercer componente es el comportamiento del sector privado no bancario, que es el objetivo de la presente investigación.

El comportamiento del sector privado comprende la lorma en que éste integra sus activos monetarios y cómo, a la vez, combina éstos con activos financieros 0 activos reales. En este campo, la trayectoria del

1. La base monetaria representa los pasivos netos (numerario más reservas obligatorias bancarias) de un banco central. Por el lado de las fuentes, la base monelaria es igual a la suma de los activos externos netos, créditos al gobierno y el sector bancario. Lo que debe quedar claro es que los activos monelarios del sector bancario y privado son los pasivos monetarios de la autoridad monetaria. Para una explicación más amplia, véase GLOWER (1986, 1988). 
sector privado no bancario se mide por la relación entre el numerario ( $\mathrm{N}$ = electivo en manos del público) y los depósitos a la vista (D) en el sistema financiero (N/D). No exisle razón, a priori, para saber exactamente cual será la combinación por la cual oplará el sector privado. No obstante, la evidencia empírica arroja fuertes indicios de que el comportamiento del consumidor en cuanto a este fenómeno es prediciblemente estable; de hecho, dicha estabilidad permite a la autoridad monetaria llevar a cabo sus obligaciones, plasmadas en la política monelaria.

En la medida en que un país supera los obstáculos del atraso economico y el nivel de ingreso aumenta, se puede discernir que la relación numerario/depósilos (N/D) disminuye. En la literatura económica este fenómeno es conocido como "prolundización linanciera". De lo anterior, se puede inferir que el desarrollo económico lambién involucra una modernización en los mercados monelarios y crediticios.

Así, a este indicador no se le puede restar importancia como un barómetro del desarrollo económico (GLOWER, 1985). La explicación de lo anterior es relativamente sencilla, ya que, al acelerarse el proceso de desarrollo, ésle es acompanado por el desarrollo del sislema financiero. A la vez, el sector privado conlía mas en el sistema financiero y opla por colocar sus fondos ociosos en depósilos a la vista o a plazo. Esta tendencia contribuye a un aumento del denominador $y$, por consiguiente, a una reducción de la razón N/D.

Como se podrá colegir, la razón numerario/depósitos desempena un papel muy imporlante en la teoría económica. Por un lado, N/D es un determinante fundamental del proceso de oferta monetaria ( $y$ algunos leóricos sostienen que su importancia es aún mayor, ya que dicha variable es considera un eslabón clave del comportamiento global de una economía de mercado, que contribuye directamente al ciclo económico). Por otro lado, N/D puede senalar importantes aspectos relacionados con el desarrollo de un país. A lo largo de nuestro análisis, se tendrá en mente el papel dual de la variable $N / D$. Se recalca que la bifuncionalidad de la variable bajo esludio constituye un signiticativo punto leórico con ramificaciones múltiples, dentro de las cuales sobresale la profunda relación entre los aspeclos monetarios en una economia subcapitalizada.

Con el enfoque dual como guía, en esta investigación se pretende cuantificar e interpretar el comportamiento de la relación entre el numerario y los depósitos a la visla (N/D) en El Salvador durante el periodo 1957-1988. En esos anos, El Salvador fue sacudido por diferentes sucesos. Se podrian mencionar el conflicto bélico con la hermana Republica de Honduras (1969), el inusitado aumento del precio del calé en 1976.77 y las reformas estruclurales de 1980 . A priori, seria sorprendente que el 
comporlamiento de los agentes económicos en cuanto a la relación N/D no hubiera sido afectado por esos hechos.

En paises como El Salvador, en donde existe un exiguo margen de sustitución enlre activos monetarios y aclivos financieros, todo suceso inesperado, en el plano político, económico o social, conduce a una sustitución entre activos monelarios y activos reales (joyería, bienes, raices, etc). Ese tipo de restricción afecla la razón N/D; sin embargo, la dirección del cambio no se puede saber de antemano, ya que el público puede oplar por cambiar sus depósilos por activos reales o por activos externos, o bien puede aumentar su líquidez (numerario), como medida precauloria para hacer frente a algún suceso inesperado. En olras palabras, en un pais subcapitalizado, los aclivos liquidos bien pueden ser el único tipo de aclivo financiero, por su atractiva caracteristica de inmediata liquidez en un mundo incierto.

\section{Aspectos teoricos}

Se ha Iralado de apuntalar que el comportamiento de la razón N/D es muy importante; sin embargo, en general la literatura sobre el tema, en los paises subcapitalizados es muy limitada y lo es aún más en los paises centroamericanos. Dentro de las obras realizadas merecen ser mencionadas las de Wiliord para México (1977), Khatkhate, Galbis y Villanueva para Venezuela (1980) y Dadkhah y Mookerjee para la India (1988). Dichos trabajos parten del postulado de que todo lenómeno económico es alectado por dos fuerzas generales: el ingreso y los precios.

Asi, en estas investigaciones se establece que en los paises atrasados la relación entre la razón N/D y el ingreso es negativa. Por su parte la relación entre los precios (inflación) y la razón N/D es positiva, por lo que se debe deducir que exisle una significativa suslitución entre activos monetarios y activos reales. A ese respeclo, es imperativo senalar que la especilicación apropiada en cuanto a la variable precio es la tasa de interés y que la relación entre éste y la razón N/D es negativa. En este caso, la tasa de interés representa el costo de oporlunidad de los aclivos liquidos y la inflación es un sucedáneo del coslo de oporlunidad de la tenencia de activos reales. Para propósitos práclicos, sin embargo, la dilerencia mencionada es imposible de aislar.

En un país en vías de desarrollo, al contrario de un pais industrializado, la relación entre el ingreso y N/D es negativa, como se mencionó anteriormente. Además, el lento desarrollo de los mercados de capitales, 0 incluso su inexistencia, excluye la posibilidad de una alta elasticidad de sustitución entre aclivos monelarios y activos financieros; es más probable que la sustitución se dé entre activos monetarios inlernos y activos 
financieros externos o activos reales.

Con la intención de captar la sustitución entre activos financiaros domésticos y activos financieros exlernos, es usual introducir como variable explicativa el costo del dinero en el mercado con el cual el país subcapitalizado mantiene sus mayores vínculos comerciales. De existir algún tipo de suslitución, la variable será signilicativa y tendrá signo positivo. Estos son algunos de los aspectos que hacen que el esludio de la razón N/D en un país subdesarrollado sea lodavía más importante y merecedora de invesligación.

En términos generales, la oferta monetaria $(\mathrm{OM})$ es el producto de la base monetaria $(B M)$ y el multiplicador monetario $(\mu){ }^{2}$ esio es:

(i) $\mathrm{OM}=\mathrm{BM} \mu$

(ii) $\mathrm{BM}=\mathrm{A}+\mathrm{G}+\mathrm{B}$

(iii) $\mu=[(1+(N / D)] /[(N / D)+r+e)$

por lo que se obliene,

(iv) $O M=(A+G+B)\{[(1+(N / D)] /(N / D)+r+e]\}$

donde $\mathrm{OM}$ es la oferta monetaria; $\mathrm{BM}$ es la base monetaria; $\mu$, el multiplicador monetario; $A$ los activos externos netos: $G$ el crédito neto al gobierno; $B$ el crédito nelo al sector bancario: ${ }^{3} N / D$ la razón numeración depósilos a la vista, determinada por el sector privado; e, el exceso nelo de reservas bancarias, que responde al comportamiento de los inlermediarios financieros; y $r$, la proporción de reservas obligatorias, determinada por la autoridad monetaria.

En las ecuaciones (iii) y (iv), se puede observar la importancia de la razón N/D para el proceso de la oferta monelaria; es más, es claro que

2. En un trabajo realizado para el Centro de Estudios Monetarios Latinoamericanos en México (GLOWER, 1986) enfoqué el problema desde el punto de vista de la autoridad y como ésla, a su vez, trata de controlar la oferta monetaria por medio de la variación de la base monetaria, en el supuesto de que el multiplicador es empiricamente predecible; consecuentemente, el presente estudio enloca un componente del multiplicador monelario y en cierla forma puede ser considerado como una extensión de aquel trabajo, con miras a afianzar aún más los resultados empiricos obtenidos.

3. Un modelo completo de determinación de la olenta monetaria exigiria una ecuación de comportamiento para el sector bancario; dicha ecuación debe tomar la diferencial entre la lasa de interés del mercado y la lasa de descuento, asi como la relación entre dicha diferencial y las reservas bancarias. 
un aumento en N/D disminuye el valor del mulliplicador monelario y, casi simultáneamente, reduce la oferta monelaria (ceteris paribus). Consiguientemente, es obvia la repercusión de una variación de la razón N/D sobre la efeclividad y alcance de la política monelaria.

Diferenles factores influyen en la estructura de la cartera del público no bancario, de la cual la razón N/D es un indicador exacto. La estructura en sí es una función de la fortuna total del consumidor, y de la distribución del ingreso y la fortuna en loda la economia. Vinculado con ello, la distribución de los saldos monelarios entre numerario y depósitos depende del grado de confianza que haya logrado granjearse el sistema financiero; mientras mayor es dicho grado, menor es la razón N/D. Por su parte, la inestabilidad, política o económica generalmente, pero no siempre, aumentará la razón. También, el propio proceso de desarrollo económico, el cual conlleva la modernización del sector rural, conduce a que se incrementen las sucursales bancarias en lodos los rincones de la economia, contribuyendo asi a un aumento de los depósitos y tinalmente a una reducción de N/D.

Finalmente, dentro de este entoque, se recalca que el aspecto importante que no debe perderse de visla es el papel que juega la razón N/D para elucidar el proceso de transterencia de fondos tinancieros, de los que tienen capacidad de ahorrar hacia los que tienen capacidad de inverlir produclivamente. Con ello no se trata de menoscabar los papeles Iradicionales del dinero (medio de pago, elc.), sino más bien de realzar la primordial función desarrollista de los mecanismos e instrumentos monetarios, para superar el alraso económico.

Ante dicho entoque, es menester hacer hincapié en que el dinero es un agente activo en el proceso de desarrollo, y es responsabilidad fundamental de la autoridad monetaria asegurar el papel del dinero como agente activo y como aclivo financiero en dicho proceso. Ello se puede lograr no por los carninos táciles de abaralar el dinero, sino fortaleciendo los canales mecanismos e instiluciones por medio de las cuales las transferencias de londos y las transacciones puedan realizarse a un coslo menor. Después de lodo, el desarrollo económico implica un sostenido nivel general de crecimiento de la capacidad de producir bienes y ocio. Los aclivos monetarios representan el pivole en esle proceso.

\section{El modelo}

Con el propósito de asentar que el comportamiento de la razón N/D no es aleatorio y es de hecho un parámelro de la vida cotidiana de una economía atrasada, se estimará inicialmente un modelo autorregresivo, en su forma más rudimenlaria; así, 


$$
N / D=\theta(N / D)_{-1}
$$

o lo que es lo mismo:

(vi) $\quad(N / D)-(N / D)_{-1}=\epsilon$

La leoria económica sef́ala que una especificación correcta de la demanda de aclivos líquidos debe incluir un efecto de ingreso, un efecto de precio relativo y un electo de precio absoluto (como vector); de esta forma, la razón N/D se especificará:

$$
\begin{aligned}
& N / D=\theta(y, \pi, i) \\
& d y<0 ; d \pi>0 ; d i<0
\end{aligned}
$$

donde y es el ingreso nominal o real*; $\pi$, la tasa de inflación; e i, la lasa de interés inlerna.

Sin embargo, cabe apuntar que la poca bondad empírica de la serie de tasas de interés en EI Salvador no permite su utilización en investigaciones de indole econométrica. Dada esta restricción, debemos aceplar la serie de precios absolulos (inflación) como un sucedáneo apropiado para caplar el eleclo que corresponde a dicha variable; indudablemente, no podremos separar el electo de precio relalivo del efecto de precio absoluto, pero esa limitación solamente ejerce un efecto marginal para el proposito que esla investigación prelende alcanzar.

Además, el subdesarrollo de El Salvador en cuanto a sus mercados monetarios y crediticios, lo que implica la poca elasticidad de sustitución entre aclivos internos, y el control que tradicionalmente ha ejercido la auloridad monetaria sobre la lasa de interés (pasiva y activa), conduce a que la lasa de interés no sea el indicador apropiado del costo de oportunidad de los activos monetarios; es más, existe un número mayor de razones contundentes para suponer que la lasa de inflación, anle las limilaciones mencionadas, constituye un mejor indicador global del coslo de oportunidad.

4. La especificación de una ecuación de demanda de activos líquidos exige que la variable ingreso se especilique en términos reales; sin embargo, considerando que en éste trabajo se utilizan dos componentes de los activos liquidos y el enfoque es una proporción, el utilizar el ingreso nominal como variable explicativa no constituye un abuso metodológico, ni implica ningún tipo de premisa implicita, (ilusión monetaria, etc). No obstante, se presentan resultados econométricos que también incluyen el ingreso real como variable explicativa. 
En relación con el argumenlo anlerior y considerando que los agentes económicos, en un pais en desarrollo, atrontan serias restricciones en el proceso de sustitución de activos financieros doméslicos, es dable suponer que dichos agentes económicos optan por la suslitución entre activos monelarios internos y aclivos financieros externos (inclusive activos líquidos - monetarios- externos). Para caplar esla posibilidad, se eslimará una ecuación que incluya el costo de oportunidad de la lenencia de activos liquidos externos. Como sucedáneo de este costo de oportunidad, se utilizará el rendimiento de los "valores federales" de Estados Unidos, que tienen un periodo de vigencia de seis meses (T-Bill). Esta variable también puede indicar si la "luga de capitales" responde a una diferencial entre el rendimiento interno y el externo de la tenencia de aclivos líquidos. ${ }^{5}$

Es teóricamente aceplado que el rendimiento de dicho activo financiero (T-Bill) representa la lasa de rendimiento "sin riesgo" y por ende es razonable suponer que ésta desempefia el papel de lasa de equilibrio, a cuyo nivel tienden todas las demás tasas de interés. Bajo ese enfoque, es de esperar que la relación entre la razón N/D y la lasa de interés externa sea positiva; ello se deduce del hecho de que al incrementar dicha tasa, los agentes económicos sustituirán depósitos internos por depósilos externos $y$, al reducirse los primeros, por lo general se reduce el denominador de la razón N/D. Así, pues, se estimará la siguiente ecuación:

$$
\begin{aligned}
& N / D=\theta\left(y, \pi, i^{*}\right) \\
& \mathrm{di}^{*}>0
\end{aligned}
$$

La definición de las variables " $\pi$ " e " $y$ " es la misma que en las ecuaciones anteriores $\mathrm{e} \mathrm{i}^{*}$ es el rendimiento de los valores federales (seis meses, T-Bill) de Eslados Unidos.

5. En este contexto, el concepto de "fuga de capital" podrla ser equivoco, ya que el proceso de acumulación de riqueza en los paises subdesarroliados podría incluir, como un aspecto normal, la adquisición de activos linancieros externos. Por ejemplo, existen indicios empíricos de que los mercados crediticios engendran economias de escala, las cuales se maníliestan en un mayor rendimiento (excluida la prima de riesgo); consecuentemente, las $\theta \infty 0$ nomlas de escala, el riesgo y la inflación, conducen en forma normal y rulinaria a la "fuga de capital", en tiempos normales. Asl, la covarianza de los rendimientos de los activos monelarios en los palses atrasados, exige que, en equilibrio, las carteras financieras incluyan activos externos. (GLOWER, $1987)$. 
Partiendo de la razonable premisa de que la razón N/D capla el comportamiento del sector privado no bancario dentro del proceso de determinación de la oferta monetaria, es dable pensar que sucesos exógenos afectarán el valor cuantitalivo de la razón que aqui se investiga. En particular, este lipo de factor exógeno puede afectar el valor estimado de $N / D$, creando un desplazamiento de la curva estimada o un quiebre (ruptura) en la pendiente de la misma. Por este molivo, se puede hablar en forma hipotética de que factores exógenos, como por ejemplo la reforma bancaria de 1980, han afeclado el valor de largo plazo de la razón N/D. Siguiendo ese lineamiento, se estimará, por medios econométricos ordinarios, una ecuación que incorpore una variable cualitativa que caple algún cambio estadísticamente significativo en el valor de N/D. Así,

$$
\text { (ix) } \quad \mathrm{N} / \mathrm{D}=\quad \begin{aligned}
& \theta\left(y, \pi, \mathrm{i}^{*} \mathrm{D}\right) \\
& \mathrm{dD}<0
\end{aligned}
$$

donde $D$ es la variable ficticia cualitativa que capta el cambio estructural exógeno.

\section{La experiencla de EI Salvador}

Por definición, el medio circulante es igual al numerario $(\mathrm{N})$ más los depósitos a la vista (D). En la Gráfica 1, se puede observar como se ha desarrollado el medio circulante en El Salvador duranle el período estudiado. Es nolable como el medio circulante empieza a tener una mayor variabilidad a partir del año 1972; en ese año, se derrumbó el sistema monetario de Bretlon Woods, en vigencia durante todo el periodo de la posiguerra. Es posible que la mayor variabilidad del medio circulante en El Salvador y en todos los países de América Latina desde ese ano no sea lortuito.

Durante los afios 1957-1972, la politica económica salvadoreña tenia como objelivos un crecimiento estable y sostenido, basado en un tipo de cambio fijo y en un crecimiento de la masa monelaria congruente con las tendencias reales de la economía. No se puede descartar la premisa de que la estabilidad monelaria internacional, garantizada por Bretton woods ( $y$ por ende la menor variabilidad en la tasa de interés mundial), facilitaba el manejo de la politica monetaria en El Salvador y en olros paises con similares parámetros económicos. En lérminos más específi-

6. Para una explicación más amplia, véase Carlos J. Glower y Joel Cervantes, América Latina y el sistema monetario internacional. Cuaderno de Investigación No. 9 Centro de Estudios Monelarios Latinoamericanos: México, 1996. 
Gráfica 1

El Salvador: 1957-1990

Medio circulante

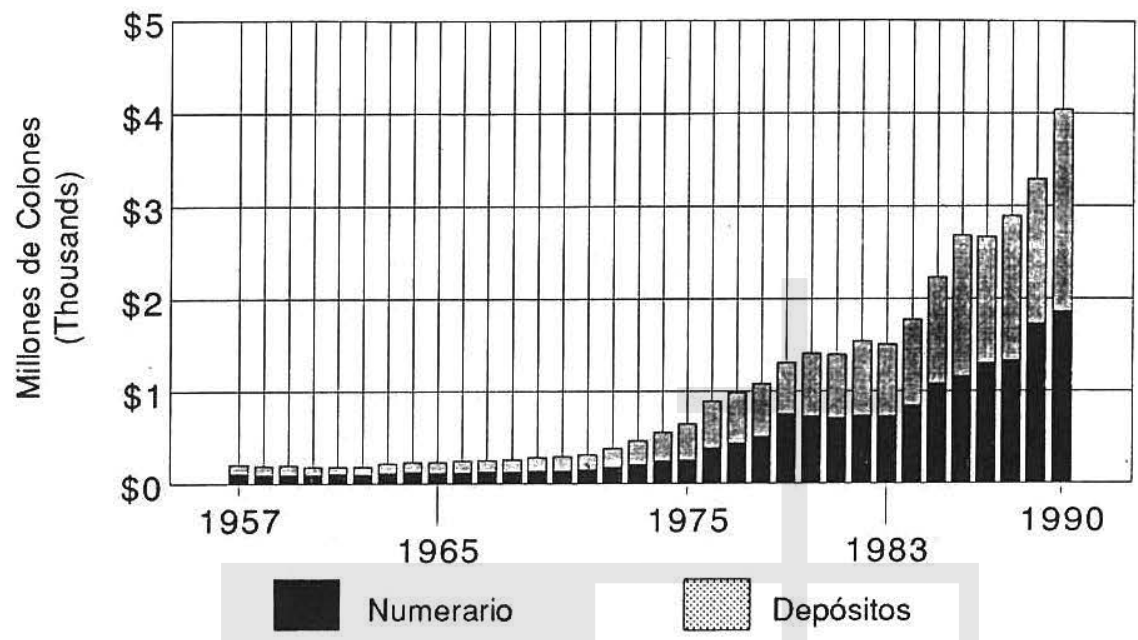

cos, la tasa de inflación fue minima y los saldos externos fueron relativamente saludables. Además, el sislema financiero demostró su pujanza por medio de la trayectoria declinante de la razón N/D, como se puede apreciar en la Gráfica 2.

A medida que la economia internacional abandonaba los lipos de cambio fijos, y la inllación mundial se volvia casi permanente al igual que la mayor variabilidad de la lasa de interés externa, el manejo de la politica económica en paises como El Salvador se dificulta aún más, pese a la supuesta mayor liberlad monetaria que implica un tipo de cambio menos rígido. Así, a partir de 1972, la autoridad monetaria salvadoreña fue perdiendo la capacidad de controlar el acervo monetario, dada la restricción que impone el tipo de cambio lijo; lundamental en el proceso también fue la poca voluntad de encarar el choque petrolero de 1973 con las medidas económicas apropiadas.

Desde otro punto de vista, y en términos mas especílicos, como se puede observar en la gráfica 2, el comportamiento de la razón N/D manliene un mismo palrón, salvo en 1960 y 1976. Cabe hacer énfasis que en estos dos años sucedieron eventos muy importantes. En el primero, se dió un cambio inesperado de gobierno, el cual agudizó la incertidumbre y marcó las pautas económicas. En el segundo, 1976, el precio internacio- 
Gráfica 2

El Salvador: 1957-1990

Comportamiento monetario

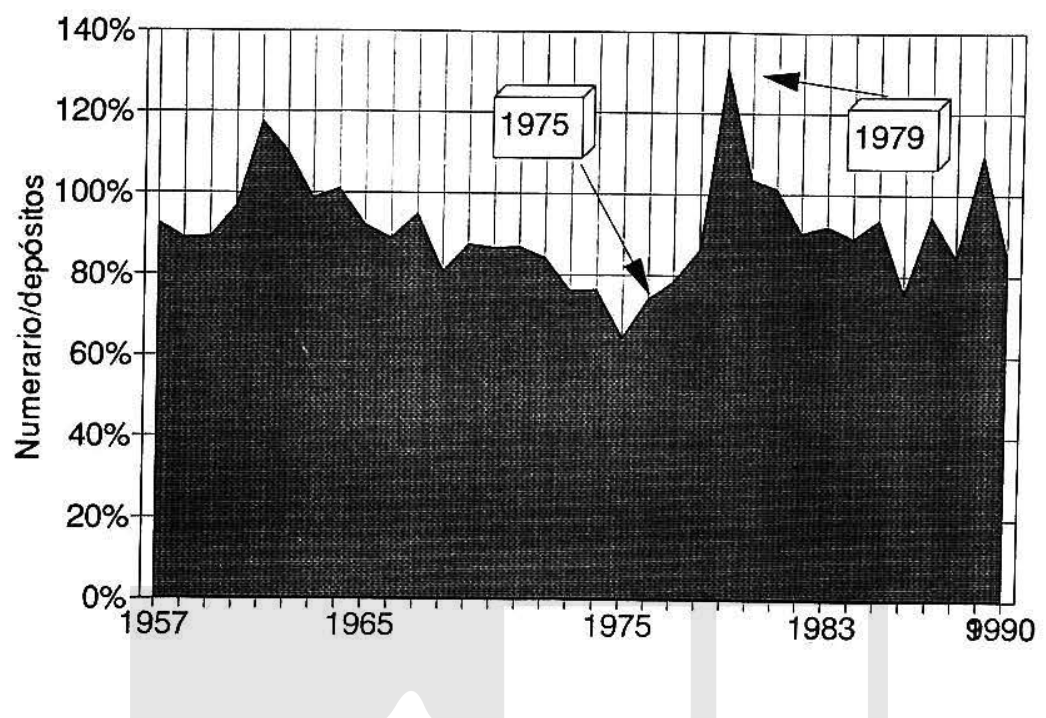

nal del calé se siluó en niveles nunca antes registrados. El flujo de dinero externo fue inusitado y rebasó todos los programas monetarios puestos en práclica. (Véase Banco Central de Reserva, 1979).

No obstante, el auge solamente duró dos anos y la razón N/D aceleró su crecimiento. Este hecho empírico es muy significativo. No se puede descartar la hipólesis de que los agentes económicos ya advertian en 1978 el desequilibrio económico-social que se avecinaba o que ya cundía. Cuando se produjo la ruptura inslitucional en 1979, la razón N/D alcanzo su mayor nivel durante todo el periodo bajo estudio.?

A partir de esta fecha (1979-88), se puede aseverar que la política económica se desvinculó de su meta de mantener un tipo de cambio tijo y/o congruente con el diseno general de la politica económica. La devaluación de 1986 no sólo no fue inesperada, sino que se implanló con varios an̂os de retraso. En anos anleriores, 1957-1978, la política económica se conformaba bajo un cuidadoso equilibrio entre la inversión públi-

7. En 1989 la razón N/D alcanzó el nivel 1.10, reduciéndose a 0.85 en 1990 . El último trimestre del año 1989 es recordado por la inlensidad del contlicto bélico, en el cual la ciudad capital se llenó de incertidumbre por las acciones de las tropas rebeldes. De alli, el aumento en la tendencia de la variable aqul estudiada. 
ca y privada, con miras a asegurar un crecimiento estable y sostenido, ante la restricción que imponia el régimen cambiario vigente.

Sin embargo, la puesta en práctica de los lineamientos generales de la política económica durante ese periodo no siempre se realizó bajo criterios sensatos o técnicos. Por ejemplo. los aspectos liscales de la política económica generalmente apoyaban los aspectos monetarios de la misma pero, desafortunadamente, el enfoque se orientaba al corto plazo, haciendo caso omiso de los importantes parámetros de largo plazo. Por ende y por ejemplo, la estructura impositiva era inelástica, lo cual era necesario para apoyar los estuerzos desarrollistas, pero no era necesariamente 10 apropiado para mantener una trayectoria de sano crecimiemto de largo plazo. De esta manera, la poca congruencia de la política económica entre el corto y el largo plazo, engendró una propensión a los déficit fiscales y por tanto, a un tipo especílico de linanciamiento público que, dada la inexistencia de un mercado de activos financieros de largo plazo, constituye una carga adicional, en detrimento de la formación de capital. El vínculo en los mercados financieros entre el corto y el largo plazo carecla de ancla. El riesgo se habia convertido en incertidumbre, categoría no sujela a la medición.

En resumen, durante el periodo 1957-1977, el plan desarrollista salvadorena se caracterizó por ciertas fortalezas, particularmente en el campo monetario y el industrial. Empero, esos logros no fueron suficientes para aminorar el desequilibrio económico-social, el cual exigia una mayor congruencia entre la política linanciera macroeconómica y la politica orientada a los sectores reales, y que consecuentemente desembocó en la elapa que ha vivido el pais. "En el periodo 1980:90 la razón N/D ha .mostrado una variabilidad mayor que en años anleriores, aunque relativamente ha vuelto a su tendencia, $\theta$ indudablemente ha incorporado el nuevo y mayor nivel de riesgo y la consecuente nueva conslelación de precios relativos. (En la Gráfica 3, se presenta, con propósilos comparalivos, el comportamiento de N/D en México durante 1957-1988. Nótese que los valores attos ocurren durante los afios en que la moneda ha sido oficialmente devaluada).

\section{Resultados empiricos}

Las observaciones ulilizadas en el presente análisis son anuales. Los datos recabados se obtuvieron de Estadísticas Financieras Internacionales (FMI) y Mermoria del Banco Central de Reserva de El Salvador. El periodo

8. En otro trabajo he desarrollado la idea de que el plan modernizante salvadoreño nació huétano, por éste y otros lactores conexos; véase GLOWER (1989). 
Gráfica 3

México: 1957-1988

Comportamiento monetario

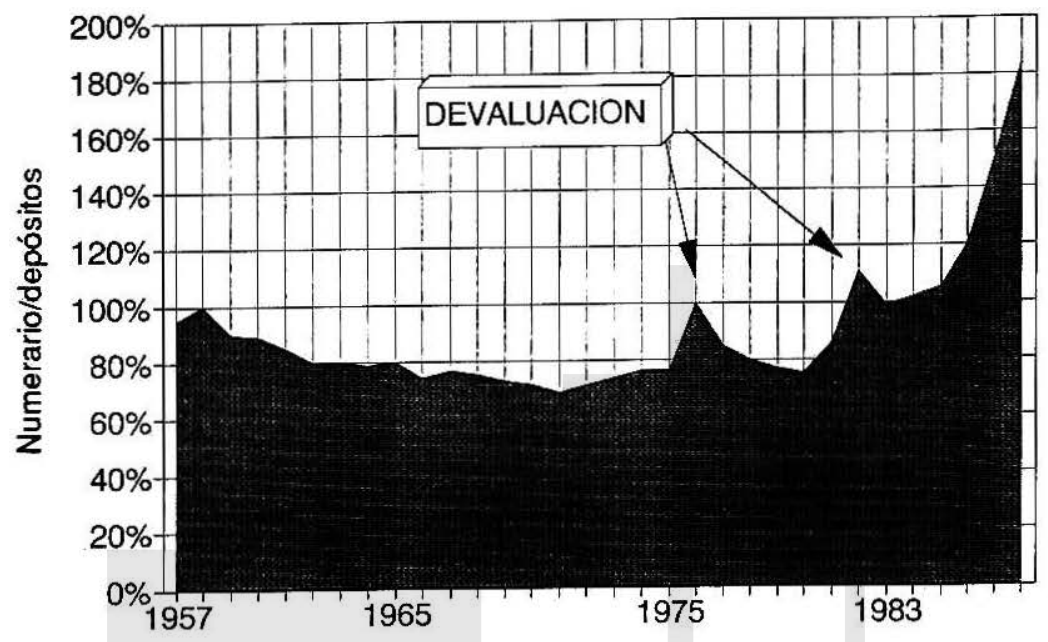

analizado parte del af́o 1957 por las limitaciones que impuso la disponibilidad de datos; cabe señalar que antes de esa fecha las series monetarias no siempre separan los depósitos a la vista de los depósitos a plazo y por lo tanto no son comparables con la variable que aqui se investiga.

Por otro lado, la forma funcional de las estimaciones es logarítmica, ya que la transformación Box-Cox arrojó valores delta $(\delta)$ cercanos a cero para todos los casos. Asimismo, con la excepción de la ecuación (1), todas las estimaciones han sido corregidas con el procedimiento de Cochrane-Orcultt, por los problemas de autocorrelación positiva, lo que puede indicar problemas de medición y no necesariamente de especificación de la ecuaciones.

Antes de presentar los resultados empíricos, sería conveniente establecer como un hecho empírico que la categoria analítica representada por la razón N/D tiene contrapartida en el plano económico y que su comportamiento no es aleatorio. Por lo tanto:

(1) $\mathrm{N} / \mathrm{D}=-0.06+0.97 \mathrm{~N} / \mathrm{D}_{-1}$

$$
R^{2}=0.98 ; F=1,276 ; \text { S.E.E. }=0.02
$$


En la ecuación estimada, se puede observar que la relación de la variable, ano con ano, es sumamente estrecha, alcanzando un coelicienle explicativo del $98 \%$ y por lo tanlo no sería prematuro decir que la razón N/D no es aleatoria. ${ }^{\circ}$

A continuación se presentan los resultados de la regresión que incluye solamente el electo de ingreso y el efecto de precio, el cual combina el efecto relativo y el absoluto. Como se puede apreciar, las estimaciones no son tan veraces como se desearia, por lo que no se puede descartar la posibilidad de que el efecto de precio relalivo debe de incluirse en lorma explicita. Lo anterior posiblemente lo está senalando el reducido valor del estadístico $F$ en ambas ecuaciones. Por su parte, el bajo valor explicativo de la ecuación $\left(R^{2}\right)$ y de las variables (valor $t$, entre paréntesis) demuestra la poca confiabilidad de esta especificación. Al incluir el electo ingreso y el electo precio absoluto, se obliene:

$$
\begin{aligned}
& \text { (2a) }(\mathrm{N} / \mathrm{D})=0.61-0.19 \mathrm{yn}-0.21 \pi \\
& \text { (2.1) (1.8) } \\
& \mathrm{A}^{2}=0.13 ; \mathrm{F}=2.06 ; \text { S. E. E.: } 0.13 \\
& \text { (2b) }(\mathrm{N} / \mathrm{D})=1.3-\frac{.19 \mathrm{yr}}{(1.9)}-\frac{.04 \pi}{(1.0)} \\
& \mathrm{R}^{2} \quad=0.12 ; \mathrm{F}=1.92 ; \text { S.E.E.: } 0.13
\end{aligned}
$$

[yn: ingreso nominal; yr: ingreso real; $\pi$ : tasa de inflación]

Los resullados anteriores nos llevan a considerar la necesidad de incluir el efecto de precio relativo, en nuestro caso, dicho efecto se abarcará por medio de la tasa de inlerés externa (i.e, T-Bill de E.U.A. a seis meses). La introducción de ésta variable propicia, además, una medición preliminar del movimiento de recursos financieros al exterior. Los resultados son los siguientes:

$$
\text { (3a) } \begin{aligned}
(\mathrm{N} / \mathrm{D})= & 0.99-\underset{(2.4)}{0.30} \mathrm{yn}+\underset{(2.3)}{0.29 \pi+\underset{(1.3)}{0.1} \mathrm{i}^{*}} \\
\mathrm{R}^{2}= & 0.18 ; \mathrm{F}=2.25 ; \text { S.E.E.: } 0.13 \mathrm{i}^{*}
\end{aligned}
$$

9. Se hace hincapié en que lodas las ecuaciones fueron estimadas en forma logarítmica, por lo que los coeficienles calculados también representan la dimensión de semielasticidad entre dichas variables y la variable dependien. te. 
(3b)

$$
\left(N / D=2.4-0.32 y r+0.1 \pi+0.13 i^{*}\right.
$$

$\mathrm{R}^{2}=0.18 ; \mathrm{F}=2.08 ;$ S.E.E.: 0.13

$\left[i^{*}=\right.$ rendimiento de T-Bill E.U. A. a seis meses $]$

La variable ingreso, como se esperaba, es significativa (95\%) y tiene el signo apropiado en ambas especificaciones. La variable precio absoluto es significaliva en la especificación nominal, pero no en la real; la variable sucedánea del precio relativo no es significativa.

Un vislazo rápido a la Gráfica 2, nos demuestra el drástico cambio en el valor de la razón N/D en 1976. De hecho, el cambio es lan pronunciado que es posible que la curva $N / D$ se haya desplazado conjuntamente con un cambio fundamental en su pendiente. A continuación, se harán dos estimaciones que nos permitirán aislar el tipo de cambio esinuclural; primeramente se presentan los resultados suponiendo un desplazamiento de la curva y posteriormente se presentan los resultados suponiendo un cambio en la pendiente de la curva. Se recalca que un desplazamien10 indica un cambio en los parámetros (nivel de ingreso, precio de olros activos/bienes, guslos, etc), mientras que un cambio en la pendiente indica una modificación en el precio de mantener saldos monelarios, lo que significa un movimiento a lo largo de la curva.

Al especificar la ecuación con la variable sucedánea de un desplazamiento, el valor de los estadísticos explicativos mejora sustancialmente. Por ejemplo, el rango de confiabilidad del estadístico $\mathrm{F}$, para las cuatro estimaciones, es de $88 \%-94 \%$. En particular, las variables ingreso nominal y real son significativas en el nivel de $99 \%$ de conliabilidad, y conlinúan manteniendo el signo apropiado. La variable precio (absoluto) es signiticativa solamenle con la variable ingreso nominal. La variable cualitativa que capla el cambio estructural (desplazamiento) es significativa $(95 \%)$ en ambas especiticaciones; como se esperaba por que los sucesos ocurridos en 1960, 1976 y 1979 -en la especificación de la ecuación solamente se ha lomado el año 1976-, su signo es positivo e indica un aumento en la razón $N / D$, lo que es compatible con lo que postula la teoría. Los resultados oblenidos son los que se senalan a continuación:

$$
\begin{aligned}
& (\mathrm{N} / \mathrm{D})=1.9-0.41 \mathrm{yn}+0.29 \pi+0.09 \mathrm{i}^{*}+0.26 \mathrm{DD} \\
& \text { (3.4) }
\end{aligned}
$$


$(4 \mathrm{aa})(\mathrm{N} / \mathrm{D})=1.6-0.32 \mathrm{yn}+0.21 \pi+0.26 \mathrm{DD}$

(4b) $(\mathrm{N} / \mathrm{D})=3.4-\underset{(.3 .0)}{0.4 \mathrm{yr}}-\underset{(1.2)}{0.06 \pi}+\underset{(1.4)}{0.11 \mathrm{i}^{*}}+\underset{(2.2)}{0.21 \mathrm{DD}}$

$\mathrm{R}^{2} \quad=0.30 ; \mathrm{F}=4.1$; S.E.E.: 0.12

$R^{2} \quad=0.31 ; F=2.98 ;$ S.E.E.: 0.12

(4bb) $(\mathrm{N} / \mathrm{D})=2.5-0.28 \mathrm{yr}-0.06 \pi+0.23 \mathrm{DD}$

(2.9)

$\mathrm{R}^{2} \quad=.26 ; \mathrm{F}: 3.25 ;$ S.E.E.: .13

[ DD: variable ficticia cualitativa que capta desplazamiento]

La variable cualitativa para el caso salvadorefio es muy singular. Como se sehaló anteriormente, el cambio drástico en N/D obedeció a Ires evenlos independientes, que ejercieron el mismo tipo de efecto sobre la variable bajo estudio. El acelerado y extraordinario flujo de divisas (dinero externo) en 1976 y 1977, no pudo ser captado con la misma rapidez por el sistema financiero, causando un aumento de N/D; conjuntamente, la inestabilidad política causó una disminución en los depósitos $y$, por ende, un aumento en N/D. Adicionalmente, el aumento en N/D posiblemente refleje el desaceleramiento del crecimiento del ingreso, como también un cambio en las preterencias del público en cuanto a la composición de su cartera monetaria, en respuesta a los nuevos parámetros.

Para el caso de una variación de la pendiente, los resultados empiricos son positivos. El cambio en el precio de mantener saldos monelarios posiblemente ocurrió por la via de la dilerencial de rendimienlos esperados entre aclivos internos y externos, agudizada por la vertiginosa alteración del nivel de riesgo e inflación, lo cual siempre alecta fuerte y decididamente el precio real de un activo financiero.

Los resultados que se basan en la variable que capla la ruptura en la pendiente de la curva marcan indicios interesantes. En ambas especificaciones, la variable ingreso se torna menos signilicativa, convirtiéndose la variable precio absoluto en la más importante. Como primera aproximación, la teoría económica nos enseña que una ruplura en la pendiente se explica por los precios y no por el ingreso. (En cuanlo a un desplazamiento, es la variable ingreso la que debe sobresalir; nuestros resultados con la variable DD con(irman esta alirmación). De ahi que se pueda aseverar, en forma preliminar, que el electo de precio lue levemente 
mayor que el efeclo ingreso, ya que el valor del esladístico $F$ logra el nivel de confiabilidad de $99 \%$ al ulilizar la variable que capta el quiebre de pendiente, confirmando una especificación mas apropiada. Los resultados empíricos son los siguientes:

$$
\begin{aligned}
& \text { (5a) }(\mathrm{N} / \mathrm{D})=0.21-0.13 \mathrm{yn}+0.20 \pi-0.03 \mathrm{i}^{*}+0.89 \mathrm{DP}
\end{aligned}
$$

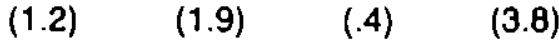

$$
\begin{aligned}
& \mathrm{A}^{2} \quad=0.46 ; \mathrm{F}=5.73 ; \text { S.E.E.: } 0.10 \\
& \text { (5aa) }(\mathrm{N} / \mathrm{D})=0.34-0.17 \mathrm{yn}+0.09 \pi+0.87 \mathrm{DP} \\
& \text { (2.3) (2.6) (4.3) } \\
& \mathrm{R}^{2} \quad=0.47 ; \mathrm{F}=8.3 ; \text { S.E.E.: } 0.11 \\
& \text { (5b) } \quad(\mathrm{N} / \mathrm{D})=0.99-0.16 \mathrm{yr}+0.09 \pi-0.01 \mathrm{i}^{*}+0.88 \mathrm{DP} \\
& \begin{array}{llll}
(1.4) \quad(2.4) \quad(.1) \quad \text { (3.8) }
\end{array} \\
& \mathrm{R}^{2} \quad=0.47 ; \mathrm{F}=6.1 \text {; S.E.E.: } 0.11 \\
& (5 \mathrm{bb})(\mathrm{N} / \mathrm{D})=1.1-0.16 \mathrm{yr}+0.22 \pi+0.85 \mathrm{DP} \\
& \text { (2.1) (2.4) } \\
& \mathrm{R}^{2} \quad=0.46 ; \mathrm{F}=7.9 ; \text { S.E.E.: } 0.10
\end{aligned}
$$

[DP: variable cualilativa que capla cambio en pendiente]

El valor de los esladislicos ha comprobado que el cambio de valor de la razón N/D en El Salvador obedeció a los drásticos (e inesperados) cambios en el ingreso y en los precios: las dos variables cualitativas son significativas. En visla de ello a continuación se presenlan los resullados de las eslimaciones que incluyen ambas variables cualitativas, tanto para el ingreso real como para el ingreso nominal. Se debe destacar que, en una investigación similar para 16 países latinoamericanos durante el periodo 1950-1971, el valor estimado de la variable ingreso fue de -0.27 , muy cercano a nuestro valor. (Véase Vogel y Buser, 1976) (Se admite que nuestra especificación fuerza un lanlo el mélodo; sin embargo, se presenta por los indicios que ofrece). Los resultados son los siguientes:

$$
\begin{aligned}
& \text { (6a) } N / D=1.3-0.24 y n+0.16 \pi-0.08 i^{*}+0.35 D D+1.1 D P \\
& \text { (3.0) (2.2) (1.6) (5.6) } \\
& R^{2}=0.76 ; F=16.2 ; \text { S.E.E.: } 0.07 \\
& \text { (6b) } \mathrm{N} / \mathrm{D}=2.1-\underset{(2.7)}{0.22 \mathrm{yr}}-\underset{(1.1)}{0.04 \pi}-\underset{(1.3)}{0.08 \mathrm{i}^{*}}+\underset{(5.3)}{0.33 \mathrm{DD}+1.1 \mathrm{DP}}
\end{aligned}
$$




\section{Resumen}

En esla investigación se ha logrado eslabler que en El Salvador el sector privado no bancario liene un comportamiento predecible, en un nivel empírico aceplable, particularmente para propósitos de programación monelaria y que además, distribuye sus saldos monetarios con base a los siguientes parámetros: 1) Variación del ingreso; 2) Cambios de los precios relativos; 3) Cambios de la lasa de inflación; 4) Sucesos extraeconómicos inesperados.

Como postula la leoría económica, el nivel de ingreso es una variable muy importanle para explicar el comportamienlo de N/D. La teoria va aún mas alla, pues predice que en un pais subcapitalizado la relación es negativa, ya que, a medida que el ingreso aumenta, los agentes economicos aprovechan la economia de escala y se vuelven mas eficientes en la administración de sus activos monetarios. Ambos postulados fueron confirmados para El Salvador. El nivel de confianza que se obtuvo, rebasó el 99 por cienlo.

Desde el punto de vista análitico, la separación del efecto precio absoluto del precio relativo es necesaria; sin embargo, en la práclica no es posible realizar esa distinción. A pesar de ello, nuestros resultados, son muy sugestivos. En primer lugar, la variable sucedánea de los precios relativos internos-extemos no logró sobrepasar el nivel de confiabilidad de $75 \%$; de ello, se puede aseverar, con las reservas del caso, que los agentes económicos en El Salvador no especulan en los mercados monetarios externos en cuantia significativa. ${ }^{10}$ Sin embargo, las variables cualinativas tienden a sehalar que dichos agentes sl utilizan los mercados monetarios externos, y posiblemente los financieros extemos, para enfrentar la incertidumbre y asi Iratar de reducir el riesgo que ocasiona el mismo subdesarroIlo, la ineslabilidad polílica o el mal diseno de la misma política económica.

Relacionado ello al párrafo anterior, la variable precio absoluto sí moslró cierla importancia para explicar el comporlamiento de $N / D$, particularmente si la especificación incluye la variable ingreso nominal; este resultado es congruenle con lo que postula la teorla economica. En el caso de El Salvador, es allamenle probable que un aumento en la inilación conduzca a una sustitución entre depósilos y activos reales internos,

10. Se informa que se hicieron estimaciones utilizando la tasa prelerencial (prime rate) como variable explicativa; aunque mejoraron marginalmente los resultados generales, dicha variable nunca logro ser signilicativa en el nivel de $\mathbf{9 5 \%}$. No obstante, las consideraciones anallicas obligan a utilizar la tasa de rendimiento de los valores federales de los Estados Unidos (T-Bills). 
o bien, dependiendo ello de los costos de transacción, entre depósitos domésticos y depósilos externos.

El comportamiento de la razón N/D en El Salvador demuestra una alta sensibilidad a los sucesos extraeconómicos. El grado de confianza y el valor del coeficiente de ambas variables cualitativas confirman lo anterior. Este resultado advierte a la autoridad monetaria y política sobre la secuela negativa que puede causar una polílica económica mal disefiada o la falla de consenso en el plano general político.

\section{Conclusiones}

El comportamiento de las variables monetarias en El Salvador duranle el período 1957-1988, no registró las anomalias que alectaron a otros paises latinoamericanos durante el mismo periodo. A grandes rasgos, se podría alirmar que los ańos 1957-1972 representan la "época dorada", en la cual los aspectos fiscales y monetarios de la política económica guardaron una alla congruencia con el plan general desarrollisla, basado en la sustitución de importaciones.

Sin embargo, a partir de 1972 , ya sea por razones externas, tales como el derrumbe del sistema monetario internacional o el efecto del incremento de los precios del petróleo, o por razones internas, tales como la incertidumbre en cuanto al modelo de desarrollo, el patrón económico salvadoreno comenzó a demostrar muestras de fatiga. La unidimensionalidad del proceso modernizante llevado a cabo condujo a un callejón sin salida, entre cuyos efectos sobresalieron los crecientes estrangulamienlos, que desbordaban con mayor magnilud sobre el plano social.

En el úllimo decenio, 1979-88, los sucesos sociales y políticos rebasaron por complelo el plano económico: la política económica propiamente dicha perdió su definición en el quehacer económico del país. Característico de lo anterior lue el inlausto proceso que concluyó con la devaluación de 1986. Con ello no se pretende rechazar la devaluación; más bien, se recalca que una polílica económica sólida y congruente nunca desemboca en una devaluación de 100 por cienlo.

Pese a la incertidumbre que se cierne sobre el panorama del pais, en el fuluro cercano la política económica no podrá alejarse de ciertos parámetros especíticos que tiendan a sentar las bases para un nuevo ciclo de formación de capital. Dentro de estos parámetros se destacan los siguientes.

La disciplina debe restablecerse en el sistema linanciero, para volver a granjearse la confianza del público. Este imperativo puede lograrse creando una competencia pujanle a la par de la banca estatificada. La competencia debe basarse en un rendimienlo tinanciero que sea realista, 
en comparación con el rendimiento de los activos reales.

Desde otro ángulo, la profundización financiera, que es lo que capla la razón N/D cuando ésta disminuye, puede ser restituida, siempre y cuando no se haga caso omiso de la dimensión inlernacional; sin embargo, las imperfecciones de los mercados monelarios en los paises subcapilalizados obliga a que la dimensión interna no sea olvidada. El primer paso para la restauración de una economia saludable debe originarse en la economia interna, la cual debe estar circunscrita por una conslelación de precios que rellejen la capacidad productiva del país.

Otro punlo importante es el de los precios relativos en el cual los instrumentos monetarios pueden aportar su mayor contribución. ${ }^{11}$ Como es bien sabido, existen dos tipos generales de instrumentos de polftica monelaria: los que regulan la oferta de liquidez y los que influyen sobre la demanda de ésla. En los palses como El Salvador, el énfasis ha descansado en los instrumentos que regulan la oferla. Sin embargo, podría ser opontuno el poner gradualmente en práclica los instrumentos que influyen sobre la demanda de liquidez, complementada por el uso mas racional de los instrumentos que regulan la oferla, tales como el encaje, topes de cartera, lasa de descuenlo, etcétera.

Con la mesura que exige el caso, el "instrumento" monetario regulador del lado de la demanda que se debe realzar en el programa económico es el rendimiento de los activos líquidos, sin por ello menoscabar la necesidad de mantener la distinción analítica y práctica de los conceptos de tasa pasiva y tasa acliva. En la medida en que los problemas del atraso económico sean vencidos, en la misma medida diluirá la necesidad práctica, pero insensata, de hacer la dislinción. El mercado se ocupará de ello.

Como lineamiento general, y partiendo del resultado empírico de que la relación entre N/D y el ingreso es negativa, sería recomendable hacer los ajustes necesarios en la programación financiera de la auloridad monetaria. En otras palabras, en la medida en que se restablezca la trayecioria de crecimiento económico del país, la razón N/D disminuirá, afectando inversamente el multiplicador monelario $y$ así exigiendo un mayor grado de moderación de parte de la autoridad monetaria en lo que se refiere al manejo de la base monetaria.

Por último, se subraya que lodo proyecto económico de estabilidad

11. Los instrumentos monetarios casi siempre se han utilizado para afectar el nivel absoluto de precios, con miras a ejercer un electo recesivo (Cavallo, 1977). En ese proceso, se ha olvidado por completo el extraordinario papel que puede desempeñar el sector monelario para afianzar una constelación de precios realistas, partiendo de la relación entre el rendimiento de los activos monelarios y el de los activos reales. 
debe partir sobre una base clara y especílica de lo que se debe estabilizar. El Salvador no está en capacidad de darse el lujo de aumentar gratuitamente la incertidumbre de los agentes económicos. Especificamente, un hecho singular que se ha perdido de vista ante los sucesos políticos que ha vivido el pals, en que durante los uiltimos anos, El Salvador ha sido colocado junto a la "Pandilla de los Seis": Uganda, Nicaragua, Corea del Norte, Sudan, El Salvador y Haitl (Institutional Investor; 1983, 1984, 1985, $1986,1987,1988$ ). Ante tal situación, y sus consecuencias, se impone un desafio que no se puede perder de vista. De esta manera, el reto primordial con que se enfrenta el actual gobiemo es el imperalivo de restitulr la confianza en las inslituciones, particularmente las de indole financiera. EI segundo gran reto lo constituye el diseno y la puesta en práctica de un plan económico-social congruente con la realidad que vive el país. Según ese enloque, la instrumenlación de la política económica es el barómetro sin par de la capacidad que posee un gobiemo para sobresalir en el arte de gobernar. Sin embargo, el imperativo primordial es el establecimiento de la Iranquilidad social. Esta úllima no es medida por el mercado, por lo menos al corto plazo. La incertidumbre en general es su aproximación.

\section{REFERENCIAS}

Banco Central de Reserva de El Salvador. (1979). "La afluencla extraordinarla de divisas y la politica monetaria" Boletin del CEMLA. (Julio-Agosto): 240.48.

Domingo F. Cavallo. (1977). "Stagflation effects of Monetarist stabilization policles". (Cambridge: Harvard University, Ph. D. thesls).

Karnran M. Dadkhah y R. Mookerjee. (1988). The Behavior of the Currency Deposit Ratio in India". Joumal of Developing Areas (Abril): 399-372. Maxwell Fry. (1988). Money, Interest, and Banking in Economic Development. Baltimore The Johns Hopkins University Press.

Carlos J. Glower. (1985). "La interrelación entre el ahorro intemo y el cambio estructural en América Latina: un análisis factorial". Monetaria (Abril-Junio): 153-194.

Carlos J. Glower. (1986). "Polltica monetaria y control en Centroamérlca" Monetaria (Enero-Marzo): 154

Carlos J. Glower y Joel Cervantes I. (1986). "América Latina y el slstema monetario internacional". Cuademo de Investigación CEMLA.

Carlos J. Glower. (1997). "La fuga de capinal en Centroamérica 1977-1984". Estudios Sociales Centroamericanos. (Diciembre): 53-70.

Carlos J. Glower. (1988). "Politica monetaria en Centroamerica: evolución reclente y perspectivas". Realidad Económica y Social (Julio-Agosto): 33-360.

Carlos J. Glower. (1989). "La modernización de El Salvador: una aspiración frustrada". Presencia (Enero-Marzo): 42-50.

Deena R. Khalkhate, V. G. Galbis y D.P. Villanueva. (1980). "A Money Multiplier Model for a Developing Economy: The Venezuelan Case". En W. Coats y D.R. Khatkhate, ed., Money and Monetary Policy in Less Developed Countries. New York: Pergamon Press.

Robert C. Vogel y Stephen A. Buser. (1976). "Inflation. Financial Repression and Capital Formation in Latin America". En Ronald J. McKinnon, ed., Money and Finance in Economic Growth and Development. New York: Marcel Dekker. Inc.

D. Sykes Willford. (1976). Money Policy and the Open Economy: Mexico"s Experience. New York: Praeger Publisbers. 